\title{
Der Arztbesuch als Luxus
}

\section{Daniel Schröpfer}

Dr. med., Medizinischer Direktor Stadtärztlicher Dienst Zürich, Vorstand VLSS

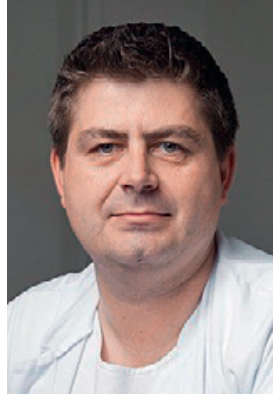

Die Gesundheitsversorgung der Schweizer Bevölkerung ist auf hohem Niveau, lese ich in vielen Studien. Im Vergleich $\mathrm{zu}$ anderen Ländern, insbesondere in Europa, sind wir Spitzenreiter. Aber sind in den Studien alle Menschen, die ärztliche Unterstützung benötigen, wirklich berücksichtigt?

Ein nicht unerheblicher Anteil an Familien, Paaren und alleinstehenden Menschen kann sich eine ärztliche Versorgung aus finanziellen Gründen nicht leisten. Viele Menschen haben Schwierigkeiten, die Krankenkassenprämien zu bezahlen, und bemühen sich um Unterstützung in Form einer Prämienverbilligung. Doch die Anspruchsvoraussetzungen dafür sind in den letzten Jahren in vielen Kantonen verschärft worden, und die gesprochenen Zuwendungen sinken deutlich. Einige Menschen unserer Gesellschaft können ihre Prämien für die Krankenkasse nur intermittierend zahlen und werden dafür je nach Wohnkanton - mit einem Eintrag in der «schwarzen Liste» «belohnt». Damit ist verbrieft, dass nur lebensnotwendige Therapien bezahlt werden. Wer entscheidet dies? Zu welch absurden Situationen dies führt, ist wiederholt der Tagespresse zu entnehmen.

Die Auswertungen des Bundes bestätigen meine Aussagen: Gemäss Daten des Bundesamtes für Statistik [1] sind die Gesundheitskosten pro Person und Monat zwischen 2010 und 2018 von CHF 662 auf 785 gestiegen.

\section{Viele Familien, Paare und alleinstehende} Menschen können sich eine ärztliche Versorgung aus finanziellen Gründen nicht leisten.

Dies entspricht einer Steigerung von knapp 20\%. Das durchschnittliche verfügbare Einkommen der Privathaushalte im gleichen Jahr betrug CHF 7069 pro Monat - wobei die Löhne von mehr als $60 \%$ aller Schweizer Haushalte unter diesem Wert liegen [2].

Ein Arztbesuch wird zu einem (fast) unerreichbaren Luxus. Provokativ ausgedrückt: Die medizinische Grundversorgung ist in der Schweiz abhängig von der sozialen Situation, insbesondere der finanziellen Situation.

Diese Entwicklung der letzten Jahre verfolge ich mit grossen «Bauchschmerzen». Die Anzahl der betroffe- nen Erwachsenen, die sich keinen Arztbesuch leisten können, steigt jährlich. Das sind Menschen, die arbeiten, eine Familie unterhalten und mitten unter uns leben. Aus meiner Sicht ergibt sich zusätzlich ein Problem bei den Heranwachsenden. Diese lernen aus dem Verhalten der Eltern respektive Bekannten, dass trotz Berufstätigkeit bei (irgendwie aushaltbaren) gesundheitlichen Beschwerden keine ärztliche Versorgung in Anspruch genommen wird. Zudem kann dadurch für

Erreichen die bisher getroffenen Massnahmen auch jene Menschen, die ihre Krankenkassenprämien nicht oder kaum zahlen können?

Kinder und Jugendliche der Eindruck entstehen, dass die Notfallstation die erste Anlaufstelle in der Gesundheitsversorgung sei - aus meiner Sicht höchst bedenkliche Entwicklungen.

Wäre es nicht an der Zeit, die Rahmenbedingungen so zu setzen, dass es allen Menschen unabhängig von ihren gesellschaftlichen Determinanten möglich ist, die medizinische Grundversorgung in Anspruch zu nehmen?

Eine Anstrengung, die sich aus meiner Sicht lohnen würde - und eine Entwicklung mit nachhaltigen Folgen für unser Gesundheitswesen darstellen würde. Es geht mir nicht darum, bisherige Veränderungen und Lösungsvorschläge zu be- oder verurteilen, doch sind diese für die zukünftigen Herausforderungen adäquat? Erreichen die bisher getroffenen Massnahmen auch jene Menschen, die ihre Krankenkassenprämien nicht oder kaum zahlen können? Zeigen geplante Massnahmen für diesen Personenkreis Wirkung?

Wir sollten uns auch die Frage stellen, wo wir in der Schweiz diesbezüglich im Hinblick auf die Millennium Development Goals (MDG) der WHO stehen.

Jede Anstrengung in Richtung einer positiven Veränderung sollte unterstützt werden. Der Arztbesuch ist kein Luxus und muss allen möglich sein.

\section{Literatur}

1 www.bfs.admin.ch/bfs/de/home/statistiken/gesundheit/ kosten-finanzierung.assetdetail.14840816.html

2 www.bfs.admin.ch/bfs/de/home/statistiken/wirtschaftlichesoziale-situation-bevoelkerung.assetdetail.14963784.html 